\title{
Syphilis manifesting as a nasopharyngeal carcinoma with cervical lymphadenopathy: A case report
}

\author{
XINBIN PAN $^{1}$, XIAODONG ZHU $^{1}$ and QINGDI QUENTIN $\mathrm{LI}^{2}$ \\ ${ }^{1}$ Department of Radiation Oncology, Cancer Hospital of Guangxi Medical University, Nanning, \\ Guangxi 530021, P.R. China; ${ }^{2}$ National Institutes of Health, Bethesda, MD 20892, USA
}

Received January 25, 2012; Accepted March 21, 2012

DOI: $10.3892 / \mathrm{etm} .2012 .535$

\begin{abstract}
The present case report describes a case of syphilitic lymphadenopathy and raises the awareness of the differential diagnosis of cervical lymphadenopathy. A 50-year-old male worker presented with a 6-month history of enlarged and growing lymph nodes in the right upper neck and a bloodtinged post-nasal drip. Physical examination showed multiple enlarged lymph nodes located in the right upper neck. On nasopharyngoscopy, a mass was found in the nasopharynx. The histopathology of both the nasopharyngeal mass and the enlarged lymph nodes revealed non-specific inflammation. Rapid plasma reagin test results (titer, 1:1280) and Treponema pallidum particle assay results (titer, 1:2560) were positive. Subsequently, a diagnosis of syphilis was confirmed clinically and serologically. The reaction after penicillin treatment further confirmed the syphilis diagnosis. Thus, syphilis should be considered as a possibility in the differential diagnosis of cervical lymphadenopathy.
\end{abstract}

\section{Introduction}

Syphilis is a sexually transmitted disease caused by the spirochete Treponema pallidum, which can invade any mucous membrane and cause lymphadenopathy. The clinical presentation can involve chancre formation, regional lymphadenopathy, widespread mucocutaneous lesions, condyloma lata, cardiovascular syphilis, neurosyphilis and syphilitic gummas, according to the natural course of the disease. Chancres and regional lymphadenopathy are common clinical manifestations of syphilis. A chancre usually begins as a single firm, non-tender,

Correspondence to: Professor Xiaodong Zhu, Department of Radiation Oncology, Cancer Hospital, Guangxi Medical University, Nanning 530021, P.R. China

E-mail: zhuxiaodong1966@yahoo.com.cn

Dr Qingdi Quentin Li, 216 Watkins Pond Blvd., Rockville, MD 20850-5622, USA

E-mail: liquenti@mail.nih.gov

Key words: syphilis, cervical lymphadenopathy, nasopharyngeal carcinoma, differential diagnosis raised, painless lesion located at a site on the penis, cervix, vaginal wall or anus; it is rarely found in the nasopharynx (1-4). Regional lymphadenopathy, a notable clinical manifestation of syphilis during its early stage, may involve any of the lymph nodes, with the inguinal nodes being most frequently involved; the disease is a rare cause of cervical adenitis.

Cervical lymphadenopathy is associated with a number of disease conditions; most often, these are tuberculosis, distant metastasis and lymphoma. Differentiation among infectious, metastatic and lymphomatous cervical lymph nodes is critical from a therapeutic viewpoint. Arriving at the correct diagnosis as early as possible is also important, as a delayed diagnosis can lead to upstaging of the malignancy, possibly making a curable lesion incurable. In South China, one of the most important diagnoses of cervical lymphadenopathy is nasopharyngeal carcinoma, which usually presents clinically as cervical lymphadenopathy, nasal blockage and epistaxis (5).

In the assessment of patients with cervical lymphadenopathy, a history and full clinical examination are essential (6). This can be supplemented by radiological diagnostic techniques such as computed tomography (CT) and magnetic resonance imaging (MRI) and by an examination under anesthesia (7,8). Further assessment with a fine-needle aspiration biopsy or an open biopsy can help to confirm or refute a diagnosis of head and neck malignancy $(9,10)$.

The present study reports a case of syphilis without chancre formation that manifested as nasopharyngeal carcinoma with enlarged cervical lymphadenopathy and a nasopharyngeal mass.

\section{Case report}

A 50-year-old male worker presented with a 6-month history of enlarged and growing lymph nodes of the right upper neck and a blood-tinged post-nasal drip. His mother had died from nasopharyngeal carcinoma 10 years earlier.

Physical examination showed multiple enlarged lymph nodes located in the right upper neck. These were painless, firm and non-tender, with the largest measuring 4x3 $2 \mathrm{~cm}$. There were no signs of genital involvement, skin eruption, or inguinal or axillary lymphadenopathy.

On nasopharyngoscopy, a mass was found in his nasopharynx and was biopsied. No lesions were noted in the oropharynx or larynx (Fig. 1A). MRI of the head and neck showed a mass in the nasopharynx and multiple lymph nodes at 
A

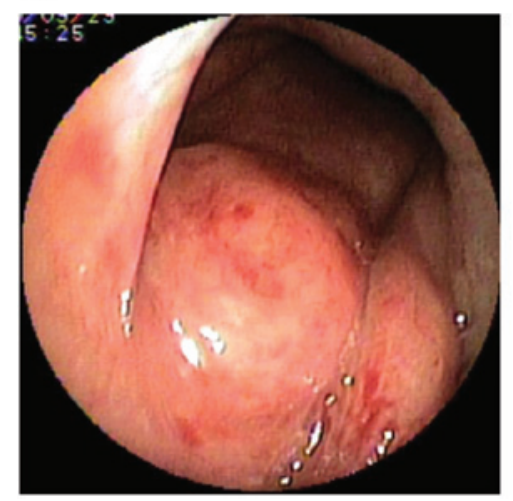

B

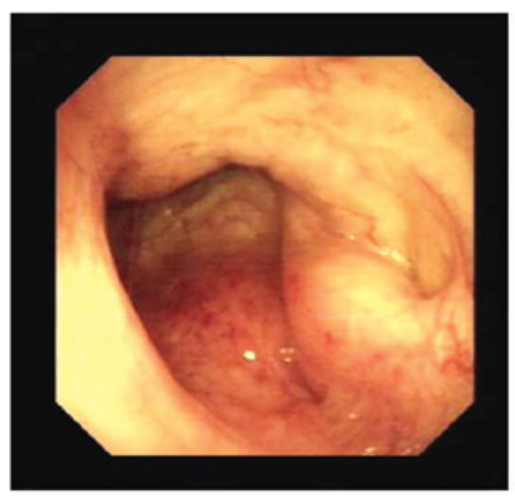

Figure 1. (A) Upon nasopharyngoscopy, a smooth pink mass was seen in the nasopharynx. (B) The mass in the nasopharynx disappeared after penicillin treatment.
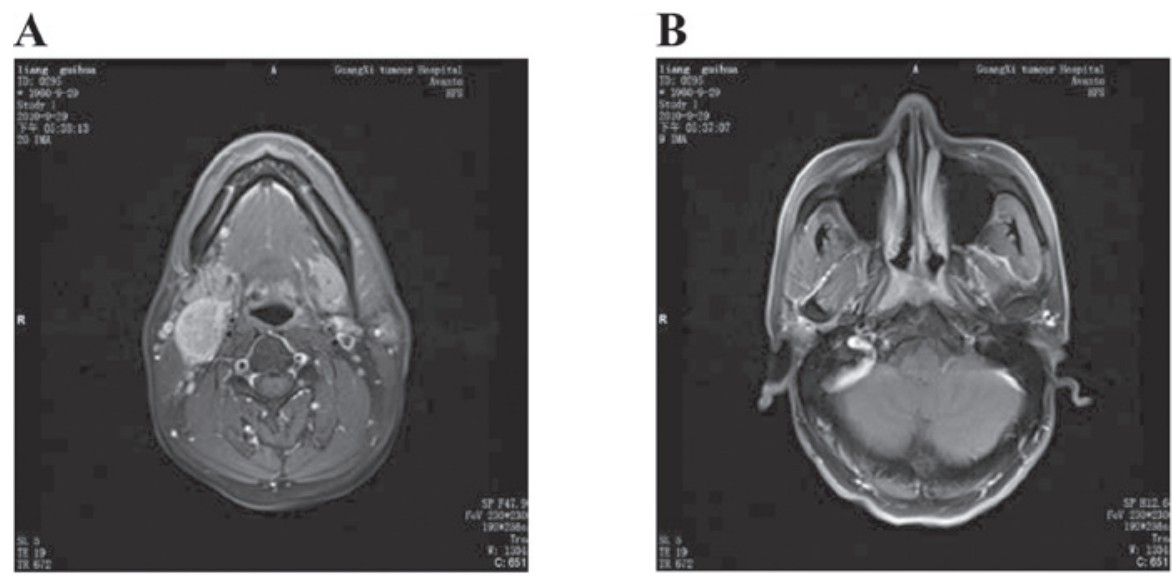

Figure 2. (A) An axial MRI scan after administration of contrast material shows an enlarged lymph node with high signal intensity. (B) The enhanced mass had invaded both pharyngeal recesses.
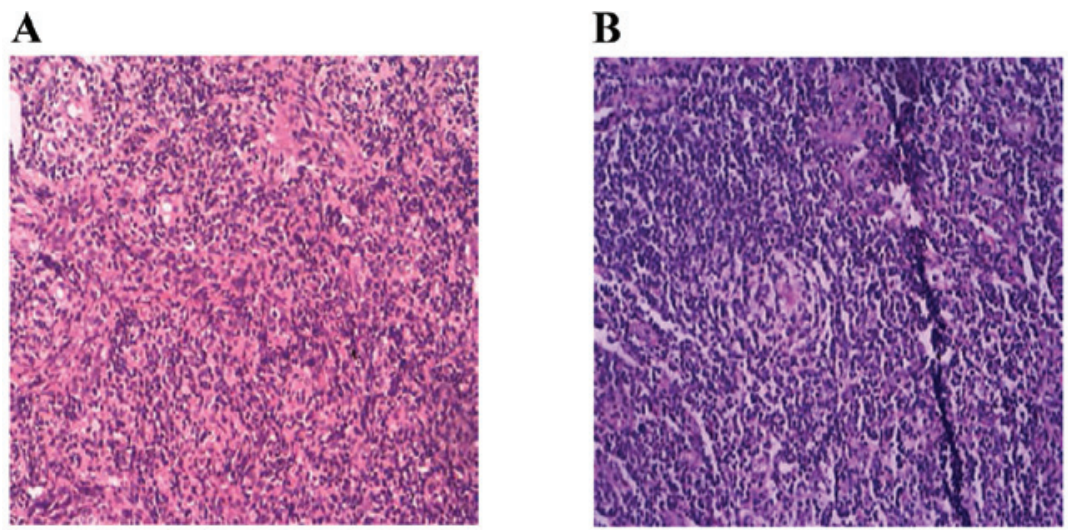

Figure 3. (A) Histopathology of the nasopharyngeal mass reveals chronic mucosal inflammation (H\&E, x100). (B) Lymphadenitis of the resected enlarged neck lymph node (H\&E, x100).

the II, III region. On T1-weighted images, the mass and lymph nodes had homogenous intensity, similar to that of muscle; high signal intensity was noted on T1-weighted enhanced images (Fig. 2). The clinical impression was nasopharyngeal carcinoma with cervical lymph node metastasis.
Histopathological examination of the nasopharyngeal mass demonstrated chronic mucosal inflammation, which was confirmed by immunohistochemistry showing that the mass was immunonegative for leukocyte common antigen and cytokeratin (Fig. 3A). Histopathological examination of the 
resected enlarged neck lymph node revealed lymphadenitis (Fig. 3B). There were no signs of nasopharyngeal carcinoma or lymphoma.

Rapid plasma reagin (RPR) test results (titer, 1:1280) and Treponema pallidum particle assay (TPPA) results (titer, 1:2560) were positive. Neither human immunodeficiency virus (HIV) nor hepatitis B virus was detected. A diagnosis of syphilis was confirmed clinically and serologically.

The patient was treated with penicillin $G$ benzathine, 24 million units in one intramuscular injection repeated at weekly intervals for 3 weeks, without a Jarisch-Herxheimer or allergic reaction. At the 12-month follow-up after the completion of treatment, a physical examination showed a significant size reduction in the lymph nodes. The nasopharynx had a normal appearance and no masses, upon nasopharyngoscopy (Fig. 1B). RPR test results were negative, but a TPPA gave positive results.

\section{Discussion}

The prevalence of syphilis has risen over the last decade (11). At the same time, syphilis, known as a great masquerader, can be difficult to diagnose because of its variable manifestations. Clinicians should be aware of the possibility of syphilitic lymphadenopathy when confronted with cervical masses, especially without chancre formation or other manifestations of syphilis.

A sexual history is important for a syphilis diagnosis, but patients often tend to conceal their sexual histories from physicians. The patient in our case did not indicate risky sexual behaviors in the initial clinical history. Further careful history-taking after TPPA and RPR testing revealed that he had experienced sexual promiscuity, although he denied oral sex.

The differential diagnosis for lymph node enlargement includes infections and neoplasms. The infectious diseases include tuberculosis, toxoplasmosis, HIV infection, catscratch disease, Lyme disease and rubella; the malignant diseases include lymphomas, lymph node metastases and carcinomas (12). Syphilitic cervical lymphadenopathy is often misdiagnosed because it is thought to be a primary or metastatic tumor. As in the present case, the manifestations of a blood-tinged post-nasal drip, growing enlarged right upper neck lymph nodes and nasopharyngeal mass were likely to lead physicians to diagnose nasopharyngeal carcinoma with lymph node metastasis. His positive family history for nasopharyngeal carcinoma and MR imaging findings both further indicated the misdiagnosis.

The use of MR to image the head and neck region has become increasingly widespread, but the efficacy of MRI in evaluating cervical lymphadenopathy has not been fully evaluated (13). In our case, both the nasopharyngeal mass and cervical lymph nodes had homogenous intensity on T1-weighted images and high signal intensity on T1-weighted enhanced images. The MRI findings strongly indicated a nasopharyngeal carcinoma diagnosis, although it is non-specific for malignancy. There is little information concerning imaging patterns of syphilitic cervical lymphadenopathy, and findings of syphilitic lymphadenopathy are limited. Thus, it is unclear how specific and/or diagnostically helpful MR images would be.
18F-fluorodeoxyglucose positron emission tomography/ computed tomography (18FDG PET/CT) may be helpful for the differential diagnosis of infections and neoplasms. However, positive findings on 18FDG PET/CT may mimic many diseases. Lymph node uptake is most often the result of malignant diseases, but 18FDG can accumulate with any inflammation, including mycobacterial disease, for example. Therefore, abnormalities on MRI and 18FDG PET/CT could be interpreted incorrectly.

We diagnosed the patient with syphilis based mainly on RPR and TPPA serologic tests, after excluding malignant diseases by histopathology. The TPPA, widely used as a confirmatory test for syphilis, is specific and has a highly positive predictive value. The RPR test is used primarily to assess the progression of syphilis or the efficacy of treatment. In our case, both the RPR and TPPA titers were high before therapy. The TPPA results remained positive, while the RPR results were negative, following penicillin $\mathrm{G}$ benzathine therapy. The titration and time courses of the RPR and TPPA titers, and the patient's clinical course strongly suggested an acquired syphilis diagnosis.

In conclusion, we report the case of a patient with syphilis manifesting as a nasopharyngeal carcinoma with cervical lymphadenopathy and a nasopharyngeal mass. Clinicians should keep syphilitic cervical lymphadenopathy in mind when making the differential diagnosis of neck masses.

\section{References}

1. Baarsma EA, Kazzaz B and Soei KI: Secondary syphilis of the tonsils. J Laryngol Otol 99: 601-603, 1985.

2. Fiumara NJ and Berg M: Primary syphilis in the oral cavity. Br J Vener Dis 50: 463-464, 1974.

3. Kleidermacher P, Vito KJ and Strome M: Otolaryngologic manifestations of acquired syphilis. Otolaryngol Head Neck Surg 119: 399-402, 1998.

4. Shimizu T, Shinogi J, Majima Y and Sakakura Y: Secondary syphilis of the tonsil. Arch Otorhinolaryngol 246: 117-120, 1989.

5. Wei WI and Sham JST: Nasopharyngeal carcinoma. Lancet 365 : 2041-2054, 2005

6. Rood SR and Johnson JT: Examination for cervical masses. Postgrad Med 71: 189-194, 1982.

7. Rumboldt Z, Gordon L, Gordon L, Bonsall R and Ackermann S: Imaging in head and neck cancer. Curr Treat Options Oncol 7: 23-34, 2006.

8. Hermans R: Head and neck cancer: how imaging predicts treatment outcome. Cancer Imaging 6: S145-S153, 2006.

9. Tandon S, Shahab R and Benton JI: Fine-needle aspiration cytology in a regional head and neck cancer center: comparison with a systemic review and meta-analysis. Head Neck 30: 1246-1252, 2008.

10. Saboorian MH and Ashfaq R: The use of fine needle aspiration biopsy in the evaluation of lymphadenopathy. Semin Diagn Pathol 18: 110-123, 2001.

11. Righarts AA, Simms I, Wallace L, Solomou M and Fenton KA: Syphilis surveillance and epidemiology in the United Kingdom. Euro Surveill 9: 21-25, 2004.

12. Damion J and Hybels RL: The neck mass. 2. Inflammatory and neoplastic causes. Postgrad Med 81: 97-103, 106-107, 1987.

13. Kaji AV, Mohuchy T and Swartz JD: Imaging of cervical lymphadenopathy. Semin Ultrasound CT MR 18: 220-249, 1997. 